\title{
THE LADM-BASED 3D UNDERGROUND UTILITY MAPPING: CASE STUDY IN SINGAPORE
}

\author{
Jingya YAN $^{1 *}$, Siow Wei JAW ${ }^{1,4,5}$, Kean Huat SOON $^{2}$, Gerhard SCHROTTER $^{3}$ \\ ${ }^{1}$ ETH Zurich, Future Cities Laboratory, Singapore-ETH Centre - Jingya.yan@ arch.ethz.ch \\ ${ }^{2}$ Singapore Land Authority, Singapore SOON_Kean_Huat@sla.gov.sg \\ ${ }^{3}$ Geomatik + Vermessung Stadt Zurich, Zurich, Switzerland Gerhard.Schrotter@zuerich.ch \\ ${ }^{4}$ Geoscience \& Digital Earth Centre (INSTeG), Research Institute for Sustainable Environment, \\ Universiti Teknologi Malaysia, Malaysia; swjaw@utm.my \\ ${ }^{5}$ Department of Geoinformation, Faculty of Built Environment and Surveying, Universiti Teknologi Malaysia, Malaysia
}

KEY WORDS: Underground Utility, 3D Data Model, Land Administration Domain Model (LADM), Ground Penetrating Radar (GPR), Utility Cadastre

\begin{abstract}
:
A digital twin can be defined as a realistic representation of something physical. To improve decision making, a reliable digital twin of the underground is required. Utilities represent a significant portion of physical assets existing underground. Most of these utilities are situated in the relatively shallow layers of the underground, up to a few meters below the surface. The reliable information on subsurface utilities has clear benefits all throughout the life cycle of state land, resulting in efficient decision-making processes, cost savings, and additional revenues for land administration professionals. However, the lack of a reliable map of subsurface utilities lead to ill-informed decisions, costly information gathering, and missed business potential. This paper will share a case study of the 3D underground utility mapping workflow from data capture to usage in Singapore. The new data is collected by the Pegasus: Stream, a mobile mapping platform which captures above and underground data using the photo, laser and ground penetrating radar technology. Based on the Land Administration Domain Model (LADM) 3D underground utility data model, the new collected data is used with the existing utility and cadastral data for land administration. This study case expects to explore the reasonable workflow of 3D underground utility mapping to provide reliable information for land administration.
\end{abstract}

\section{INTRODUCTION}

Over the years, in order to meet the many and growing needs of urban development, a lot of underground spaces have been used for the public infrastructures, such as utility lines, rail lines and roads. Complex underground infrastructure and inaccurate underground information complicates the development of underground space, the ownership of underground objects is not clear for example; the interdependency of above ground and underground has not presented directly, another example. Having said that, some countries and institutions have implemented or at least conceptualized the 3D mapping of underground utility network and their management in relation to cadastral system. The City of Zurich for instance has an own utility cadaster platform since 1999 and has set up a governance framework with the corresponding utility providers. UK, Qubec and Rotterdam begin to register underground objects for land administration.

Singapore is a city state that experiencing land scarcity, where there is a need to optimize land resources for economic and social development. The use of underground spaces in Singapore is increasingly evolving multi-layered overlapping development for transportation, utility infrastructure and storage facilities. To support the use of these underground spaces, a Singapores Underground Masterplan will unveil in the year 2019. A good spatial understanding of underground information is undoubtedly a key driver of smart planning and resilient use of the underground spaces. In order to understand the demands of underground utility data users, a workshop was organized to learn the work process and needs of land administration

\footnotetext{
${ }^{*}$ Corresponding author
}

in Singapore. This study includes four application domains, (i) land acquisition and purchase, (ii) planning and coordination, (iii) land transfer and sale, and (iv) land leasing. Before the return of land to Singapore Land Authority (SLA), the owner needs to declare everything beneath the ground including utility services. The existing setup within the underground will affect future developers design and impact on developing costs directly. Currently, the existing data sources are a hardcopy of the utility network, 2D CAD and 2D geospatial information. Without depth information, it is difficult to known whether the data represents the current situation, the possibly different as-built state, or just the as-designed state. Furthermore, the geometric accuracy and the completeness of the area often unknown. Obviously, there is an urgent demand of 3D geospatial information of underground utility and space to evaluate underground environment and support reallocation, land sales and the other applications. It is necessary to register the utility segments as the legal objects in the land administration system, which helps to identify the ownership of underground utility.

A reliable 3D digital map of utility networks is crucial for urban planners to understand the impactful aspects of the underground space. A consolidated underground utility database is backbone to connect data acquisition and usage. The aims of this paper are to design and implement a 3D utility data model for land administration based on large scale underground utility mapping in Singapore. Our previous work (Yan et al., 2019) provides a workflow of data governance includes data capture, processing, integration, storage and using. This work provides a case study of 3D utility mapping workflow from data capture to data modelling. It includes two main tasks: 
- The conceptual design of LADM-based 3D underground utility data model. In order to connect 3D modelling of underground utility to land administration, some of the attributes and values of this data model are inherited from Singapore cadastral data model and LADM (ISO 19152). The LADM is a link to build a relationship between the geometric information of utility network and land administration management. Some objects and attributes of this data model will inherit LADM and Singapore cadastral data model. Meanwhile, the geometric and spatial definition are inherited from spatial schema data model.

- Based on the 3D underground utility data model, a consolidated underground utility database will be developed. In this database, the underground utility data will be integrated with cadastral data. The underground utility data includes two parts: one is the existing data in the database of the government agency, which is as-build data in the 2D form; the other is the newly collected data by Ground Penetrating Radar (GPR).

Section 2 shows the case study of using the Pegasus: Stream, a mobile mapping platform which captures above and underground data using the photo, laser and ground penetrating radar technology in Singapore. Section 3 introduces the design of 3D underground utility data model for land administration. Based on the results of data capture by Pegasus and the data model, a study case is implemented to visualize the land administration information of underground utilities.

\section{LARGE SCALE UNDERGROUND UTILITY MAPPING: CASE STUDY IN SINGAPORE}

\subsection{Mobile based 3D Ground Penetrating Radar}

The Pegasus: Stream mobile mapping platform was deployed at the nine selected study sites in Singapore (Figure 1). This mobile mapping platform is first time deployed in South East Asia. It aims to assess the feasibility of the large volume of data capture by this platform to improve the quality of underground utility survey in provide reliable underground utility data. Eventually, this can contribute to sustainable city planning and underground land administration. This mobile mapping platform combines a Stream EM GPR and Leica Pegasus Two photo and laser scanner. The Pegasus:Stream allows the capturing of large volume underground data and imagery and point clouds of the above ground features. All the data captured by the Pegasus: Stream is geo-referenced using a combination of on-board sensors that include a GNSS receiver, an Inertial Measurement Unit (IMU), and a Distance Measurement Instrument (DMI). The technical specifications of the Stream:EM GPR are shown in Table 1.

In operation, this platform is towed behind a vehicle moving at a speed of not more than $15 \mathrm{~km} / \mathrm{h}$. This means it can theoretically cover and capture data of over tens of hectares per day. The potential of large-area data capture without interruption of traffic flows can reduce cost and time for mapping significantly. Despite this, and due to the slow speed of movement and lack of safety features on the platform itself, an auxiliary police escorting service was engaged for safety purposes. A special purpose license was acquired from LTA to allow the platform on the roads of the selected case study areas only.

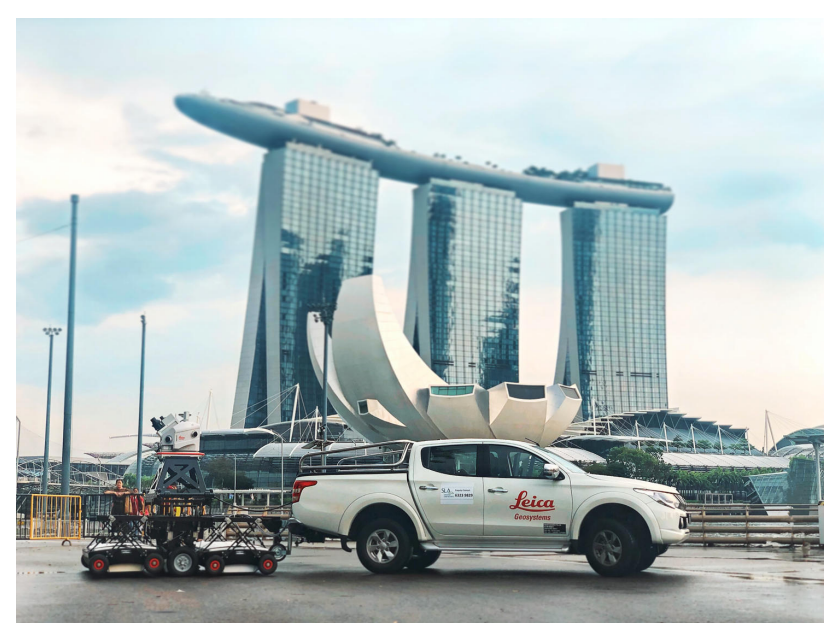

Figure 1. The Pegasus:Stream during its deployment in Singapore.

\begin{tabular}{|l|l|}
\hline Overall weight & $228 \mathrm{~kg}(500 \mathrm{lbs})$ \\
\hline Max. acquisition speed & $15 \mathrm{kph}(9 \mathrm{mph})$ \\
\hline Power consumption & $72 \mathrm{~W}$ \\
\hline Positioning & $\begin{array}{l}\text { Survey wheel and/or GPS } \\
\text { or Total Station }\end{array}$ \\
\hline $\begin{array}{l}\text { Scan Rate per Channel } \\
\text { @ } 512 \text { samples/scan) }\end{array}$ & $87 \mathrm{scans} / \mathrm{sec}$ \\
\hline Scan Interval & $\begin{array}{l}17 \mathrm{scans} / \mathrm{m} \mathrm{@} 200 \mathrm{MHz} \\
33 \mathrm{scans} / \mathrm{m} @ 600 \mathrm{MHz}\end{array}$ \\
\hline Antenna Footprint & Width $1.84 \mathrm{~m}$ \\
\hline Number of Channel & 38 \\
\hline Antenna Central Frequencies & $\begin{array}{l}200 \mathrm{MHz}(34 \text { channels }) \\
600 \mathrm{MHz}(4 \text { channels })\end{array}$ \\
\hline Antenna Spacing & $6 \mathrm{~cm}$ \\
\hline Antenna Polarization & $\begin{array}{l}\text { Horizontal (HH) and } \\
\text { Vertical (VV) }\end{array}$ \\
\hline
\end{tabular}

Table 1. Technical specifications of the Stream EM GPR.

The Stream EM GPR uses on impulse radar GPR technology. It features a large number of ground-coupled antennae, with dual frequencies $(200 \mathrm{MHz}$ and $600 \mathrm{MHz})$. Its 40 separate antennae transmit and receive in two distinct polarizations ( $\mathrm{HH}$ and $\mathrm{VV}$ ), allowing it to cover wide road surfaces and provide optimum detection for main and junction pipes at the same time. With such unique design and high numbers of antenna on board, it is expected to increase data quality and reliability of pipe detection.

\subsection{Study Sites and Case Study Primary Results}

Nine study areas were selected in different areas of Singapore. The selection includes a "virgin" practice area (near the future Canberra MRT station), relatively new residential estates (Punggol and Sengkang), relatively old residential estates (Ang Mo Kio and Toa Payoh), parts of the central business district (Raffles Boulevard and Marina Boulevard), an industrial estate (Woodlands Spectrum), and the University Town campus of the National University of Singapore. With the selection of different areas, it was attempted to investigate different subsurface contexts, e.g. different soil conditions, different utility layouts, and changes due to the progression of the subsurface over time. Figure 2 shows the distribution of the nine selected study areas.

The scanning for the nine selected study areas was conducted in the span of six days with around eight hours of work each day. This includes the time used to mount and unmount the platform 


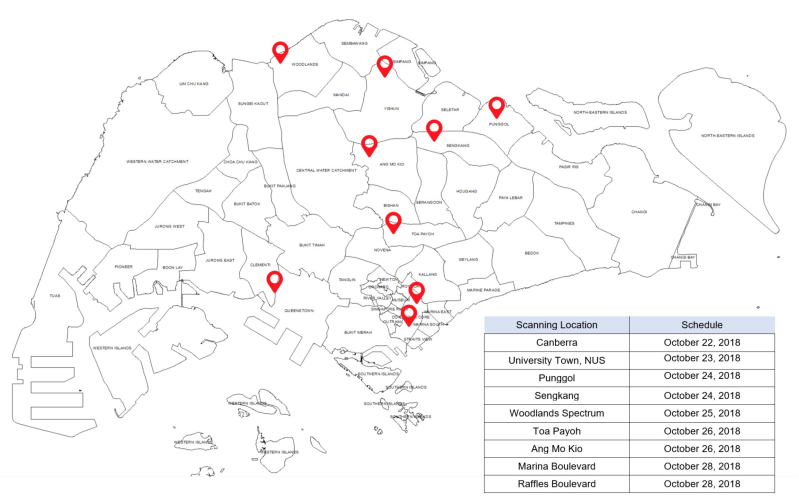

Figure 2. Map of case study areas for Pegasus:Stream.

from its transporting vehicle and the return trip to transport the platform from its storage location. For a single scan, the Stream EM can cover a swath of $1.75 \mathrm{~m}$ in width. In Singapore, the lane width is about $3.2 \mathrm{~m}$ or wider. Therefore, two scan track are typically required to cover a single lane. Over the course of six days, a total of 700GB underground data and 450GB above ground data were collected.

In this paper, we reported the most representable case study results at Toa Payoh. The main scanning route in Toa Payoh is a bidirectional loop around the selected area in between Lor 4 Toa Payoh, Lor 3 Toa Payoh, Lor 2 Toa Payoh, and Toa Payoh Central (Figure 3). The total length for one loop is about $1.8 \mathrm{~km}$. The scanning started and ended at the loading site. All roads consist of 4 lanes (2 lanes for each carriageway). The scanning at Toa Payoh proved to be challenging due to local traffic conditions and took a longer time to complete as compared to new residential estates like Punggol or Sengkang. The total time need for the scanning at Toa Payoh is 5-6 hours while the scanning at Punggol or Sengkang is about 3.5 hours only.

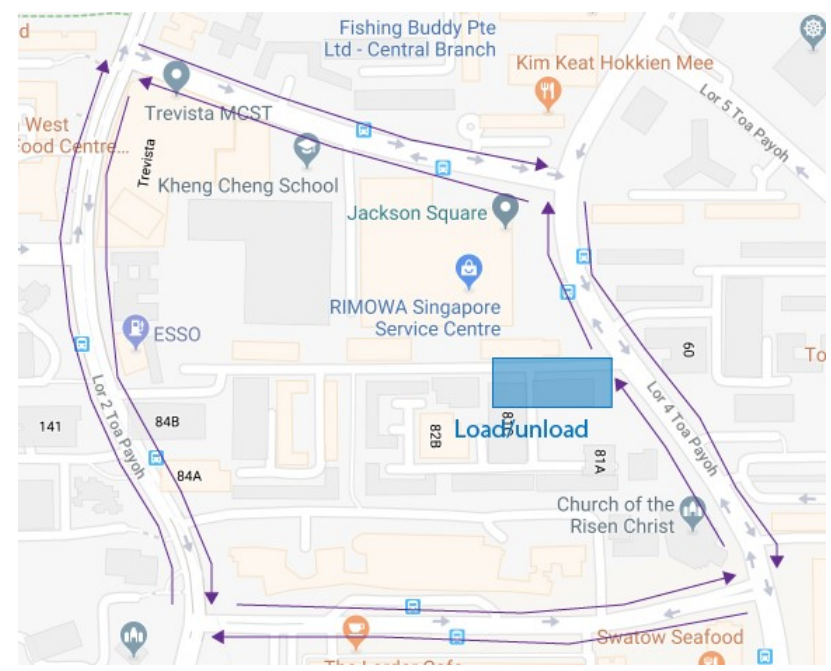

Figure 3. Toa Payoh scanning route.

All acquired data were pre-processed to enhance the quality of the radargram before further interpretation. Then, a GPR specialist interpreted and analysed the radargram (so-called B scan) to detect and extract subsurface utilities of the scanned area. The quality of the captured data is essential to determine the interpretation results (Figure 4). If the data quality is not good, it may lead to misinterpretation. After that, the data was ex- ported to CAD and GIS format for further 3D modelling and visualization.

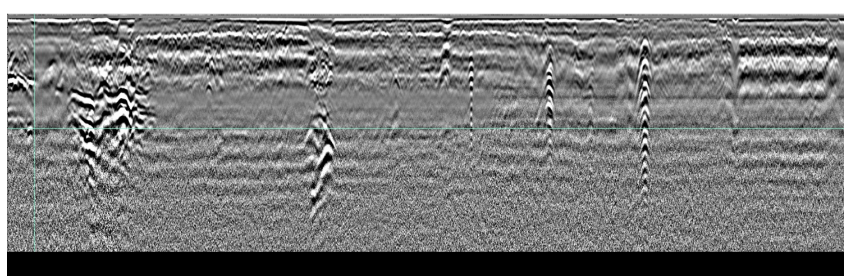

(a) Bad quality radargram

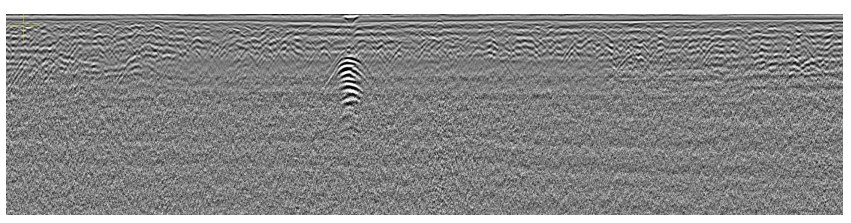

(b) Better quality radargram

Figure 4. GPR signal are highly dependent on soil condition during signal transmission.

A CAD drawing containing all captured data is shown in Figure 5. The use of a mobile mapping platform offers an enormous potential for fast subsurface data acquisition with minimal disruption to traffic. The case study in Tao Payoh, demonstrates that data captured using 3D mobile GPR can be used as a complement to existing records, especially for the mapping at old estate where those utilities were buried long ago and the utility records are incomplete or outdated. GPR seems to be paramount importance for mapping the underground utilities. However, there are others current technology that overcome the shortcoming of GPR available on the market, such as laser scanning or gyroscopic mapping. No single detection technique can detect the entire type of utilities in every location. Hence, GPR is not the only solutions for underground utility mapping, using more technologies increases the detection capability, coverage, efficiency and accuracy.

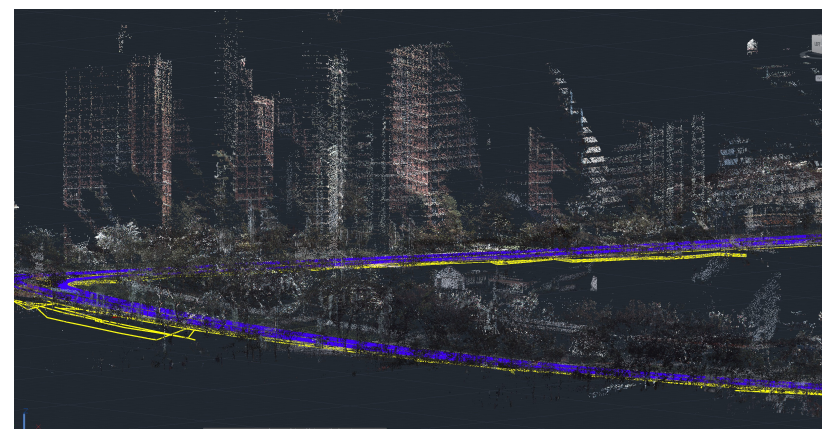

Figure 5. Example of output in CAD format for extracted utilities (yellow lines) and captured point clouds.

\section{3D UNDERGROUND UTILITY DATA MODEL}

Our previous work presents the conceptual design of 3D underground utility data model (Yan et al., 2019), which includes geometric information of utilities, data accurate management and land administration of underground utilities. This section introduces the logical design that focuses on the geometric information and land administration of underground utilities. 


\subsection{Logical Design of Data Model}

Based on the multilevel structure of utility network that is defined in our previous work (Yan et al., 2019), the utility network package should describe the geometric and spatial information of utility networks. Figure 8 shows the logical design of utility network package. The class Utility Network describes the attributes of the whole network at the top level, includes installation date and the type of utility network (e.g. water and gas). The class Utility Network Node, Utility Network Segment and Utility Network Surface are components of utility network. The class Utility Network Node and Utility Network Segment describe 2D geometric information and the other attributes of line segments and connect nodes of utility network. If the data has 3D information, the nodes and line segments are connected to the class Utility Network Surface. The class Utility Network Surface aims to describe the 3D objects of utility network, such as duct and manhole.

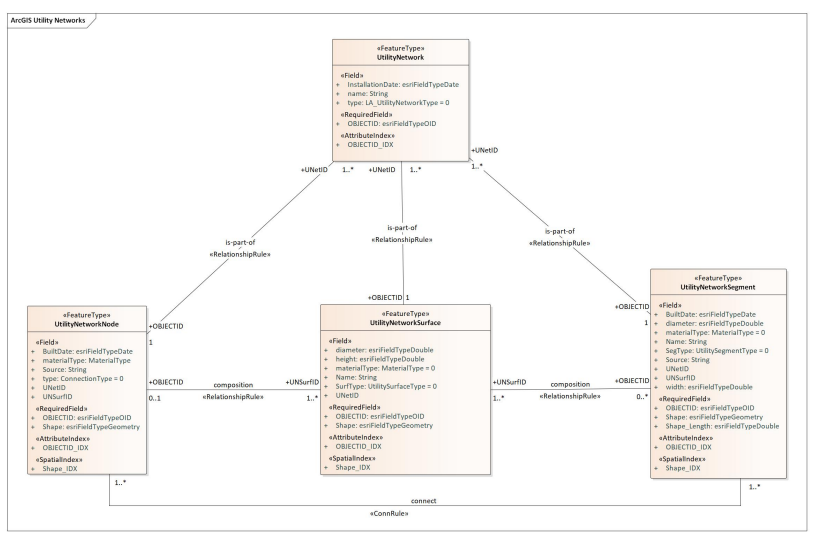

Figure 6. The logical data model of utility networks.

The class LA_UtilityNetworks plays a connection role between the LADM and utility networks. The LADM is an important legal framework to define and integrate concepts and terminology of Land Administration for 3D representations (International Standards Organization, 2012). As an international standard, the LADM provides a flexible conceptual schema from three main aspects: organizations, rights and spatial in formations. The integration of $2 \mathrm{D}$ and $3 \mathrm{D}$ information in the LADM can provide solutions for 3D Cadastre. However, LADM still lacks of information about underground utility services. To complement this, the $3 \mathrm{D}$ underground utility data model is developed to integrate underground objects with attributes from Singapore LADM, a Singapore-based LADM model customized specific for local use. Based on LADM, SLA began to develop a Singapore cadastral data model in 2014. It addresses the survey data from field to core database. Associated administrative and spatial data are included where it provides the complete picture of the cadastral database. The Singapore profile inherit LADM objects, attributes and relationships where possible, and implement new items where necessary. In the 3D underground utility data model, the class LA_UtilityNetworks connects to Utility Networks package. Meanwhile, this class connect to Singapore cadastral data model to get related land administrative information. As an important role in the land administration, cadastral parcel should be connected to the underground utility networks(Pouliot, Girard, 2016, Dner et al., 2011). Hence, the main objective of this class is to connect cadastral parcels and utilities through spatial relationships, such as contain, cross and touch. This class could support ownership management of utilities and land administration management.

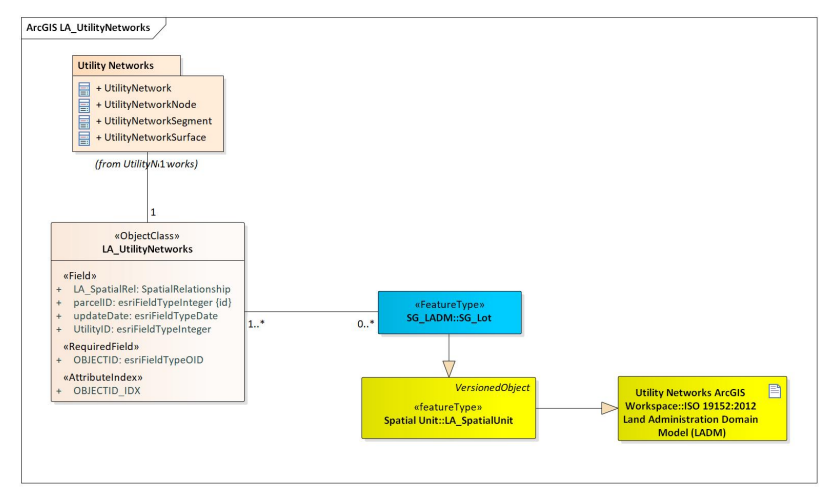

Figure 7. The connection of utility networks and land administration.

\subsection{Study Case}

3.2.1 Data Source: This study case uses three groups of datasets:

- The existing 2D utility data from GeoSpace includes water supply, sewerage, drainage, telecommunication and power grid networks.

- The 2D cadaster data from SLA. Figure 8 shows the existing utility and cadastral data in Toa Payoh.

- The new collected data using GPR, an above and underground mobile mapping platform that combines laser, photogrammetry and ground penetrating radar technology. The data were post-processed in order to obtain digital $3 \mathrm{~d}$ models of both the environment above ground environment and underground utilities in different format. After data processing, the GPR data needs to export to CAD format or GIS format with $\mathrm{x}, \mathrm{y}, \mathrm{z}$ value as points and lines for 3D data modelling and visualization. Figure 9 shows the result of GPR in GIS format in 2D view and 3D view with point cloud data that were collected by the Pegasus: Stream.

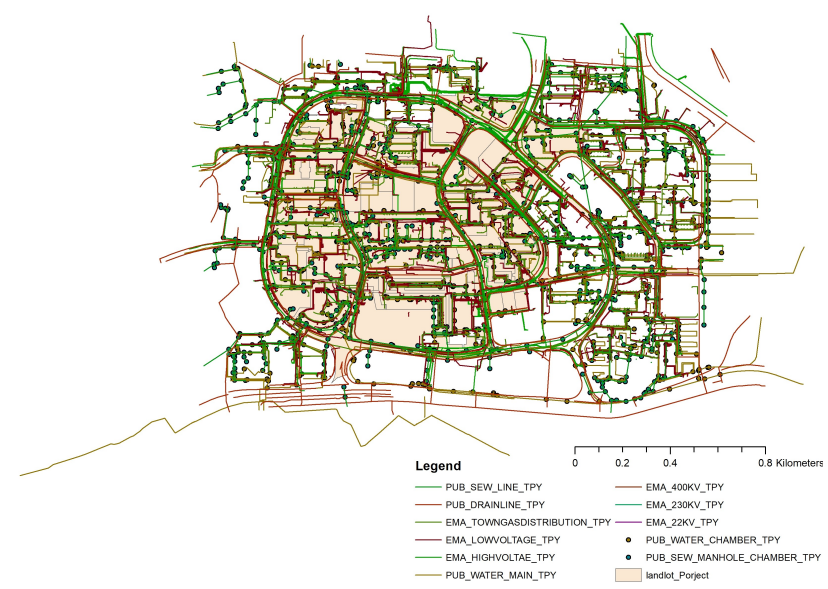

Figure 8 . The existing utility and cadaster data of Toa Payoh (Data source: Singapore Land Authority). 


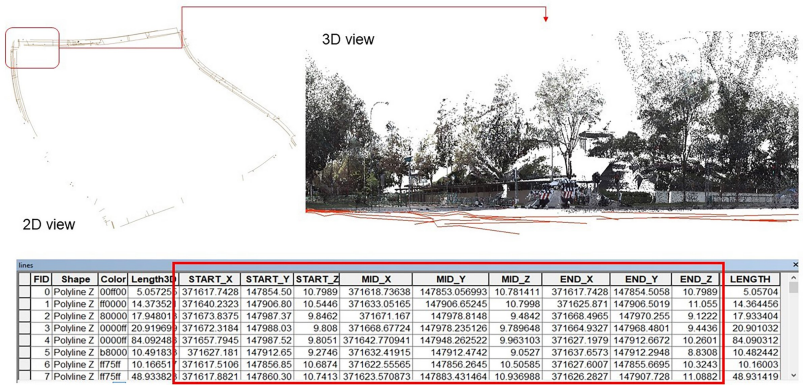

Figure 9. Results from data capture.

3.2.2 Implementation: The data model is designed by Enterprise Architect 14 using Unified Modeling Language (UML). The utility database is developed based on ArcGIS pro 2.1 . Hence, the UML data model is exported as XML document and imported to ArcGIS as File Geodatabase. Figure 11 shows the database by tree structure that includes three feature layers and three tables. This work has two steps to integrate the existing

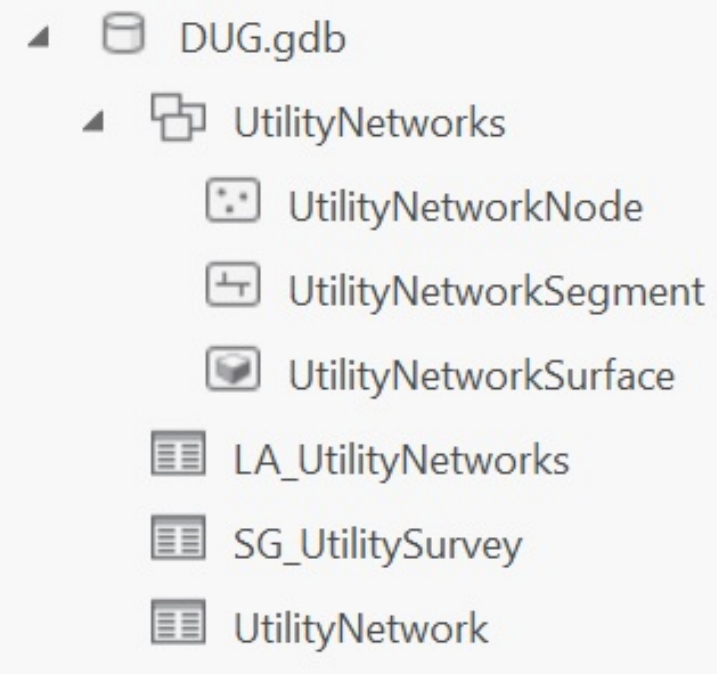

Figure 10. Underground utility database structure.

utility data and new collected data. First, in order to improve the attributes of new collected data, this experiment needs to identify new collected data from the existing utility database, which is based on spatial matching of the extracted features with other data. In order to match features, an operator overlaid the extracted features with the other data. Based on measures of spatial similarity (overlap, proximity, and visual similarity), the operator decided whether features could be positively matched. But the 2D GIS data of existing utilities imposed the following limitations: i) The lack of elevation only allows for horizontal comparison, i.e. comparison of $\mathrm{x}$ and $\mathrm{y}$ coordinates. In cases of horizontally overlapping utilities, this complicated matching and identification; ii) Identification is to take place based on horizontal overlap and proximity only. However, in many cases, the existing data did not match well with the utilities identified during data capture. This is likely due to a lack of accuracy. Additionally, it was observed that the above ground colourized point cloud data is not sufficiently detailed or suitable to confirm manhole locations, let alone identify the type of utility that is located underneath. Roughly, about $26 \%$ new collected data can be identified from the existing utility database. After identification of the required information, the new collected data is modelled in 3D. Second, the new collected data should be loaded into the new underground utility database. The points and line segments of GPR data are stored in the feature layers UtilityNetworkNode and UtilityNetworkSegment. The 3D data is stored in the UtilityNetworkSurface.

According to the underground utility database, the relationship of land parcel and underground utilities can be queried. Figure 11 shows an example of querying and visualization of cadastral information of land parcel and the underground utilities that are located within the land parcel in 2D. This example is realized by ArcPy. The simple statistic information is shown in the red box of Figure 11, which means the cadastral parcel 89 includes 40 utility nodes and 109 utility line segments in total. The detail information of cadastral parcel 89 is shown in a pop window and all the objects are selected in the map.

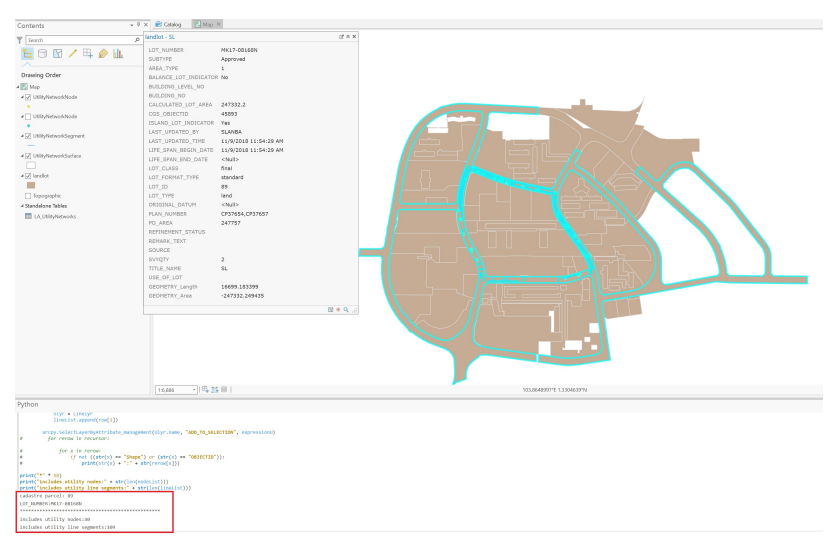

Figure 11. Visualization of underground utilities with land parcel and cadastral information.

\section{CONCLUSION}

This study case aims to test the workflow of 3D underground utility mapping from data capture to data usage for land administration. During this process, the main contributions are providing the reliable underground utility data and connect it to land administration. The use of a mobile 3D GPR platform offers enormous potential for fast subsurface data collection for the roads of large areas, with minimal disruption to traffic. From the captured data, it can be observed that subsurface utilities installed in newer areas typically appear to be better organized and planned. Most of the utilities are installed under the road reserve. The space under carriageways of Toa Payoh is occupied by many different utilities in often complex arrangements. Meanwhile, a 3D underground utility data model is designed and developed as a consolidated database. During the integration of the new collected data and the existing utility data, this work attempts to explore methods for integration of existing utility data and newly captured data. This showcase presents the connection between underground utility and land administration based on a 3D underground utility data model.

This case can be concluded that a combination of GPR and existing data alone is not sufficient to improve the accuracy of information on existing subsurface utilities. How to use the existing data? How to integrate the existing and new collected data? For the next step, resources are required to build a comprehensive 3D subsurface utility database, but also to develop a feasible approach to integrate existing and newly collected 
data. Moreover, the 3D underground utility data model connects underground utilities to cadastral parcel, which could help to identify the ownership of underground utility in land administration. However, the cadastral data is 2D in Singapore, which might not accurate to identify the spatial relationship of underground utilities and land parcel in vertical space. In the future, we will collaborate with selected agencies to evaluate and improve the 3D utility data model. Their recommendations could help to extend data model include other underground infrastructures and develop the platform of underground space management to support various applications in Singapore.

\section{ACKNOWLEDGEMENTS}

This publication has been realized as part of the project "Digital Underground: 3D Mapping of Utility Networks" at the Future Cities Laboratory, established by ETH-Zurich and Singapores National Research Foundation (NRF), and operating under the auspices of the Singapore-ETH Centre.

\section{REFERENCES}

Dner, F., Thompson, R., Stoter, J., Lemmen, C., Ploeger, H., Oosterom, P. v., Zlatanova, S., 2011. Solutions for 4D cadastre with a case study on utility networks. International Journal of Geographical Information Science, 25(7), 1173-1189. https://doi.org/10.1080/13658816.2010.520272.

International Standards Organization, 2012. ISO 19152: Geographic information Land Administration Domain Model (LADM).

Pouliot, J., Girard, P., 2016. 3d Cadastre: With or Without Subsurface Utility Network? International Federation of Surveyors (FIG), Athens, Greece. OCLC: 831214525.

Yan, J., Jaw, S. W., Soon, K. H., Wieser, A., Schrotter, G., 2019. Towards a 3D Data Model of Underground Utilities for Land Administration. https://www.preprints.org/manuscript/201904.0223/v1. 